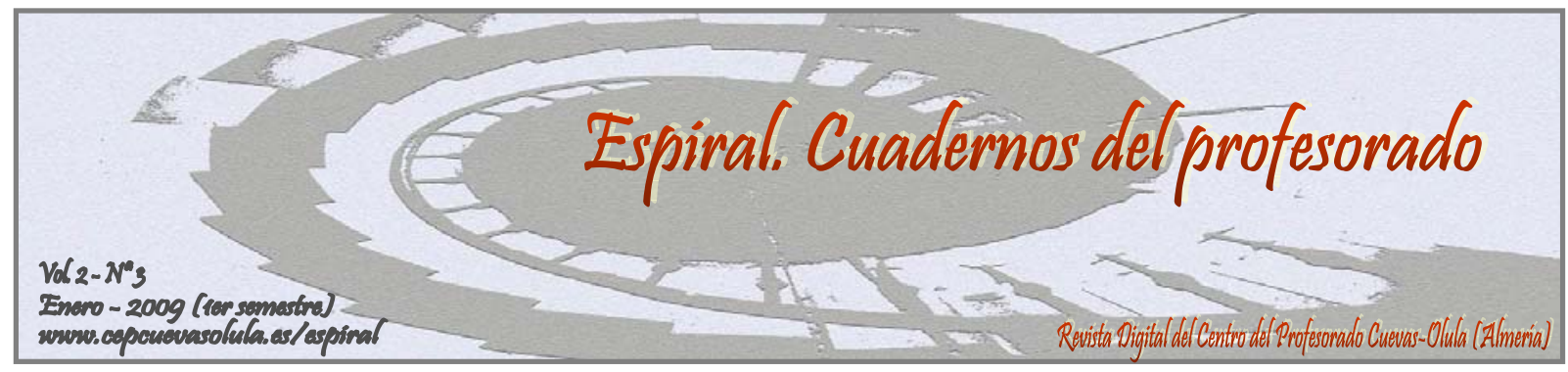

\title{
LA EDUCACIÓN FÍSICA A TRAVÉS DE UN CURRÍCULUM COMÚN EN CENTROS DE SECUNDARIA
}

\section{PHYSICAL EDUCATION THROUGH A JOINT CURRICULUM IN SECONDARY}

\section{SCHOOLS}

\section{Antonio Granero Gallegos ${ }^{(1),}$ Juan Luís López Anaya ${ }^{(2)}$, Maria de la O Godoy} Rodríguez $^{(3)}$, Juan José Núñez Carretero ${ }^{(4)}$, Marta $\mathbf{M}^{\mathrm{a}}$ Núñez Carretero ${ }^{(5)}$, Antonio Baena Extremera ${ }^{(6)}$, Patricia Ibáñez $\mathrm{Cruz}^{(7)}, \mathbf{M}^{\mathrm{a}}$ José Barrionuevo Martín $^{(2)}$, Santiago López Martínez ${ }^{(7)}$ y Félix Rodrigo Fernández ${ }^{(2)}$.

${ }^{(1)}$ Centro del Profesorado Cuevas-Olula y Universidad de Almería, Almería (España), ${ }^{(2)}$ IES El Palmeral, Vera, Almería (España), ${ }^{(3)}$ IES Velad al Hamar, Vélez Rubio, Almería (España), ${ }^{(4)}$ Licenciado en Ciencias de la Actividad Física y el Deporte, ${ }^{(5)}$ IES Azahar, Antas, Almería. (España), ${ }^{(6)}$ Facultad de Ciencias del Deporte. Universidad de Murcia (España), ${ }^{(7)}$ IES Jaroso, Cuevas del Almanzora, Almería (España)

RESUMEN: La Educación Física ha de adoptar un papel principal en esta sociedad del bienestar, del tiempo libre, del ocio. Teniendo en cuenta los resultados de diversos estudios a nivel europeo y norteamericano, en relación a la Educación Física recibida por parte de los escolares actuales, los y las componentes de este proyecto nos planteamos como objetivo principal el diseño de un currículum común del área de Educación Física entre los distintos centros de la zona, teniendo en cuenta la variedad y novedad de los contenidos a plantear en clase, intentando alejarnos de los que tradicionalmente han predominado en las sesiones de Educación Física. Se espera que un mayor y amplio número de experiencias, que parten de los intereses del alumnado, tenga unos efectos positivos en la actitud y motivación de los mismos, tanto en las clases como en un impacto positivo en la adhesión a la actividad física a lo largo de toda su vida.

Palabras clave: Educación Física, currículum, contenidos.

ABSTRACT: Physical Education has taken a leading role in this society of welfare, leisure, entertainment. Taking into account the results of various studies at European and American, in relation to physical education received by the school today, the components of this project considered the main objective of designing a curriculum area of Education Physical between different centers in the area, taking into account the variety and novelty of the content to raise in class, trying to get away from those who have traditionally dominated the sessions of Physical Education. It is expected that a greater number and broad experience, starting from the interests of students, have a positive effect on the attitude and motivation for them, both in class and in a positive impact on adherence to physical activity as throughout their lives.

Key words: Physical education, curriculum content. 

\& Rodrigo Fernández, F. (2009). La Educación Física a través de un currículum común en centros de secundaria [en línea]. Espiral. Cuadernos del Profesorado, 2(3), 55-65. Disponible en: http://www.cepcuevasolula.es/espiral.

Enviar correspondencia a: antonio.granero.ext@juntadeandalucia.es

\section{1.- INTRODUCCIÓN.}

Fomentar el aprendizaje a lo largo de toda la vida implica, ante todo, proporcionar a los jóvenes una educación completa, que abarque los conocimientos y las competencias básicas que resultan necesarias en la sociedad actual. Para contribuir a la consecución de las competencias básicas recogidas en la normativa vigente (LOE, LEA), así como para los objetivos de las distintas etapas educativas.

La Educación Física juega un papel importante, pues contempla entre sus objetivos y contenidos aspectos relacionados con el desarrollo personal y social, así como hábitos de actividad físico-deportiva y con la promoción de un estilo de vida activo entre el alumnado.

No cabe duda que las transformaciones sociales, económicas y tecnológicas han modificado los patrones de vida de cualquier parte del mundo, sobre todo a partir de la segunda mitad del pasado siglo XX, afectando principalmente a cambios en la dieta y en los patrones de actividad física. Parece claro, pues, que la sociedad actual favorece una vida diaria mucho más sedentaria que la de generaciones anteriores; que las ciudades modernas dificultan los desplazamientos a pie o en bicicleta; que las familias no permiten a los niños juegos al aire libre por percibir variados peligros en el entorno físico; que niños y niñas de todas las edades dedican cada vez más tiempo a entretenimientos de tipo pasivo tales como ver la televisión, los videojuegos o los ordenadores.

En la actualidad existe una gran preocupación por la salud en los países desarrollados, dados los elevados y crecientes porcentajes de sedentarismo y de diversas patologías asociadas al mismo, como ponen de manifiesto datos de numerosos estudios respecto al incremento de enfermedades como la obesidad, diabetes, hipertensión, etc., entre la edad infantil y juvenil (European Heart Network,
1999, 2001; 2004; Ortega et al. 2005; Serra et al., 2003).

Por ello, la orientación de la actividad físicodeportiva hacia la adquisición de hábitos saludables, así como hacia el disfrute del tiempo libre y de ocio de forma activa, con continuidad y permanencia a lo largo de la vida de la persona, deben constituir objetivos prioritarios de la Educación Física. Pero esta cuestión ha conllevado una preocupación para autoridades e investigadores, dado que la relación e influencia de la Educación Física no siempre resulta tan efectiva, sobre todo si se tienen en cuenta los anteriormente citados índices crecientes y elevados índices de sedentarismo e inactividad física en la sociedad desarrollada actual y sus consecuencias en la salud y bienestar de la persona (European Heart Network, 2001). Datos que en España, según los últimos datos de la Encuesta Nacional de Salud (2006), siguen su preocupante incremento en relación al sedentarismo y a las enfermedades asociadas al mismo.

La Educación Física ha de adoptar un papel principal en esta sociedad del bienestar, del tiempo libre, del ocio. Haciendo referencia expresa a esta área curricular y a la hora de su organización escolar, hemos de indicar que, en general, las variables de programa (objetivos, contenidos, etc.) parecen tener más importancia en la influencia sobre el alumnado que las de contexto, según la amplia literatura internacional, mostrando una mayor sensibilidad en función de los objetivos planteados por el profesor para el desarrollo de las clases, así como las formas de organización y contenidos desarrollados.

Asimismo, los resultados que ponen de manifiesto estudios actuales a nivel europeo y norteamericano, en relación a la Educación Física recibida por parte de los escolares actuales, han supuesto una preocupación por nuestra parte, teniendo en cuenta que: 
La diversión juega un papel importante para despertar una mayor motivación hacia la actividad física y su proyección en el tiempo libre (Torre, Cárdenas y García Montes, 2001).

El docente desempeña un papel fundamental en el desarrollo de la gestión de las distintas variables de programa y es el encargado de gestionar los contenidos de la mejor forma, debiendo tener en cuenta los intereses e inquietudes de su alumnado.

$\square$ Hemos de romper con la idea de la aplicabilidad nula o escasa de las clases de Educación Física.

El predominio de los contenidos tradicionalmente masculinos en las sesiones supone un rechazo por parte de las alumnas hacia la asignatura, pues los chicos mantienen el control del juego y participan más (Flintoff y Scraton, 2001). Esto tiene repercusión en el tiempo útil de la clases o el denominado tiempo de compromiso motor (Piéron, 2005), ya que los más hábiles participan más.

El alumnado dirige también sus críticas en no llegar a comprender realmente el propósito de las clases recibidas, enfatizando su reclamación en la necesidad de una Educación Física que sirva para aprender nuevas habilidades, con clases enfocadas hacia la salud y actividades de tiempo libre; asimismo, también echan en falta, para una mayor motivación, actividades de desafío y reto y que aporten nuevas experiencias (Flintoff y Scraton, 2001).

$\square$ El alumnado, en general, encuentra las sesiones aburridas y monótonas, en las que priman la repetición de actividades año tras año, con un profesorado desfasado y desvinculado de los intereses de los jóvenes de hoy en día, solicitando más variedad de actividades deportivas, un mayor nivel de desafío en las actividades de Educación Física, así como diversas actividades de aventura al aire libre (Flintoff y Scraton, 2001; MacPhail, Kirk y Eley, 2003; Mowling et al., 2004; Rikard y Banville, 2006).

De tal manera que si, como indican Luke y Sinclair (1991), los contenidos curriculares constituyen el factor más influyente en el desarrollo de las actitudes negativas hacia la Educación Física, quizá debamos prestar más atención a las críticas que se realizan hacia la asignatura y entre las que destacan aspectos relacionados con los contenidos. Asimismo, es interesante recordar la importancia de estos datos, pues como exponen Hoffman et al. (2005), Taylor et al. (1999) o Telama et al. (2005), la práctica de actividad física y recreativa en edades tempranas se asocia de forma significativa con la continuación de esta práctica de forma regular en edad adulta.

Por todo lo expuesto, los y las componentes de este grupo de trabajo nos planteamos el diseño de un currículum común del área de Educación Física entre los distintos centros de la zona, teniendo en cuenta la variedad y novedad de los contenidos a plantear en clase, intentando alejarnos de los que tradicionalmente han predominado en las sesiones de Educación Física.

\section{2.- OBJETIVOS.}

Los objetivos planteados para llevar a cabo este proyecto son los siguientes:

- Analizar los contenidos de Educación Física para secundaria.

- Conocer la opinión del alumnado referente a la Educación Física que han recibido.

- Analizar los contenidos del área que más responden a las expectativas, inquietudes, motivaciones e intereses de aprendizaje del alumnado.

- Elaborar y secuenciar un currículo común de Educación Física para la Educación Secundaria Obligatoria para ser impartido en diversos centros de la zona (y en un futuro a corto plazo llevarlo a la práctica), que suponga un incentivo entre el alumnado para la realización de actividad físicodeportiva, no sólo en el aula, sino que la integren en su vida cotidiana.

- Planificar Unidades Didácticas de contenidos novedosos, entendiendo estos como los no tradicionalmente impartidos en la Educación Física escolar y que estén en línea de los intereses analizados del alumnado, para cada uno de los cursos, teniendo en cuenta el Real Decreto 1631/2006 por el que se establece la Enseñanza Secundaria Obligatoria y que es la referencia para la estructuración de los contenidos 
específicos de cada curso, así como las opiniones e intereses de nuestros alumnos y alumnas.

- Crear un dossier con las distintas Unidades Didácticas elaboradas.

- Facilitar la enseñanza-aprendizaje de las distintas Unidades Didácticas entre los profesores y profesoras participantes en este proyecto, por parte de quien la ha propuesto y elaborado, partiendo de su experiencia en la práctica.

- Crear y participar en un foro de debate donde exponer, tratar y dar solución a la situación de nuestra materia en la actualidad y la dirección a seguir.

\section{3.- CONTENIDOS.}

- Contenidos de Educación Física para la Educación Secundaria Obligatoria.

- El currículo de Educación Física fundamentado en el Real Decreto 1631/2006.

- Análisis de intereses y motivaciones del alumnado en línea con dichos contenidos.

- Secuenciación de contenidos, por curso, del área de Educación Física.

- Elaboración de Unidades Didácticas novedosas y significativas.

- Puesta en común de las diferentes Unidades Didácticas.

- Uso de la nuevas tecnologías de la información y la comunicación para la participación en un foro virtual de debate.

\section{4.- METODOLOGÍA DE TRABAJO.}

La metodología que vamos a seguir para el desarrollo de este grupo de trabajo va a ir encaminada a la mejora de la formación y ampliación de los conocimientos teóricoprácticos del profesorado implicado y de la propia Educación Física como materia.

El punto de partida comienza por aprovechar la experiencia particular de cada uno de los integrantes del grupo como un bien común. Desde dos puntos de vista. En primer lugar, servirnos de las distintas maneras de educar por parte de los integrantes del grupo, teniendo en cuenta los años de experiencia y la progresión que experimentan los profesores y profesoras de Educación Física a lo largo de los años según el estudio realizado por Viciana (1998).

$\mathrm{Y}$ en segundo lugar, utilizar la formación particular de cada uno de los componentes del grupo, pues como dicen Castellón et al. (2001), cada uno lleva a la práctica, en mayor medida, aquello que domina. Ello conlleva poder aprovechar un trabajo ya elaborado y puesto en práctica con efectividad, añadiéndolo a nuestro trabajo diario, pues como afirman Faulkner y Reeves (2000) las experiencias del profesorado en su formación posterior, a su formación académica, incide en gran medida, en la importancia que estos dedican a los diferentes bloques de contenidos. A todo ello debemos añadir las diferentes visiones que nos proporciona un grupo de docentes que han tenido un contacto con diferentes especialidades deportivas, distinto al meramente educativo, ya sea como atletas o entrenadores, y que nos facilita la compresión entre lo que es actividad física rendimiento y lo que debe ser la Educación Física en el ámbito educativo.

Para la consecución de este proyecto vamos a utilizar, en una primera fase inicial, el método descriptivo para recabar la opinión del alumnado, así como la observación documental para el análisis e interpretación de los mismos y la elaboración de las unidades didácticas. Se llevará a cabo una revisión bibliográfica de todo aquel material que aporte una base (unos pilares) sobre los que poner en funcionamiento nuestra experiencia.

El trabajo descriptivo es el que permite obtener información sobre cuestiones tales como motivaciones, sentimientos, pensamientos, opiniones, etc. Para obtener esta información acerca de datos que forman parte de las experiencias personales de nuestros alumnos y alumnas vamos a recurrir al informe proporcionado por el propio sujeto, el alumnado en este caso.

La técnica utilizada para recabar la opinión del alumnado será la encuesta, pues ésta, como indica García Ferrando (2000), es una técnica de investigación que va a permitir explorar, describir y explicar la realidad social. Las cuestiones planteadas serán aplicadas mediante un breve cuestionario, pues se trata de uno de los instrumentos de la técnica de investigación por encuestas (Colás y Buendía, 1994; Manzano, Rojas y Fernández, 1996; Arias y Fernández, 1998); y es considerado el instrumento básico de recogida de información de la investigación social mediante encuestas (García Ferrando, 2000; Padilla, González y Pérez, 1998; Sierra Bravo, 2001). 
Tabla 1.- Comparación entre los Decretos 148/2002 y 1631/2006

\begin{tabular}{ll}
\hline \multicolumn{1}{c}{ Decreto $148 / \mathbf{2 0 0 2}$} & \multicolumn{1}{c}{ Decreto $1631 / 2006$} \\
\hline 1.- Condición Física-Salud & 1.- Condición física y salud \\
2.- Cualidades Motrices & $\ldots \ldots \ldots \ldots \ldots \ldots . . . \ldots . \ldots . \ldots$ \\
3.- El Juego y el Deporte & 2.- Juegos y deportes \\
4.- Expresión Corporal & 3.- Expresión corporal \\
5.- Actividad Física en el Medio Natural & 4.- Actividades en el medio natural \\
\hline
\end{tabular}

Una vez obtenidos, tabulados y analizados los datos, estos resultados serán la referencia sobre la que desarrollar el trabajo de elaboración de materiales curriculares, teniendo en cuenta en todo momento el Real Decreto 1631/2006 por el que se establecen las enseñanzas mínimas de la Educación Secundaria Obligatoria; Decreto 231/2007 por el que se establece la ordenación y las enseñanzas correspondientes a la educación secundaria obligatoria en Andalucía; Real Decreto 1467/2007 por el que se establece la estructura del bachillerato y se fijan sus enseñanzas mínimas; Decreto 416/2008 por el que se establece la ordenación y las enseñanzas correspondientes al bachillerato en Andalucía

Añadir, a la referencia que va a suponer el sentir de nuestros alumnos y alumnas, la importancia de nuestras propias tomas de decisión como docentes, que en este caso van a estar consensuadas como grupo, teniendo en cuenta para su elección y secuenciación, aspectos pedagógicos como es la madurez de nuestro alumnado, buscar la máxima transferencia horizontal y vertical en los contenidos, atender a necesidades motrices de los alumnos, así como a los recursos materiales, climatológicos, espaciales, etc. Y buscar la relación de las Unidades Didácticas con Efemérides, para fomentar contenidos Transversales según la legislación establecida al efecto (Baena 2006).

Por último, hemos de tener muy en cuenta los Decretos establecidos, que van a ser la guía sobre la que actuar, cerrando un triángulo sobre el que vamos a trabajar en la realización de este proyecto de "currículum-programación común" (Profesorado-Alumnado-Normativa).

Un análisis inicial del Decreto 231/2007 para la Educación Secundaria, que entra en vigor para $2^{\circ}$ y $4^{\circ}$ de la E.S.O. en este curso escolar 2008/9, revela cambios sustanciales con respecto al Decreto 148/2002 al cual ha modificado. Entre estos cambios podemos destacar.

$\square$ La supresión de uno de los bloques de contenido (tabla 1).

$\square$ Un enfoque de la condición física todavía más orientado hacia la salud.

$\square$ Una mayor especificación de los contenidos por curso.

$\square$ La incorporación de las Competencias Básicas, a las que deberemos contribuir desde nuestra materia.

Los bloques de contenidos quedan por tanto en cuatro, como podemos observar en la tabla 1.

Los contenidos constituyen otro cambio sustancial e importante, pues la normativa conlleva la introducción de una mayor especificación en los contenidos a impartir. También han sufrido modificaciones, que presentamos secuenciándolos de una manera lógica para la consecución de aprendizajes significativos en cada uno de los niveles educativos (tabla 2).

Y la incorporación, como aspecto novedoso y a destacar, de las competencias básicas, en el currículo, a las que contribuimos, de forma integrada, desde los contenidos del área de Educación Física para su desarrollo (tablas 3 y 4), teniendo en cuenta los bloques de contenidos:

B 1 - Condición física y salud;

B 2 - Juegos y deportes;

B 3 - Expresión corporal;

B 4 - Actividades en el medio natural. 
Tabla 2.- Secuenciación de contenidos en la Educación Secundaria Obligatoria

\begin{tabular}{|c|c|c|c|c|}
\hline & $1^{\circ}$ ESO & $2^{\circ}$ ESO & $3^{\circ}$ ESO & $4^{\circ}$ ESO \\
\hline \multirow{4}{*}{$\begin{array}{c}\text { Bloque } \\
1 \\
\text { Condición } \\
\text { física y salud }\end{array}$} & $\begin{array}{l}\text { - Calentamiento (Cto) } \\
\text { y actividad física (AF) }\end{array}$ & $\begin{array}{l}\text { - Objetivos y juegos } \\
\text { del calentamiento. }\end{array}$ & $\begin{array}{l}\text { - Efectos, pautas, } \\
\text { elaboración y } \\
\text { puesta en practica } \\
\text { del calentamiento } \\
\text { previo análisis de } \\
\text { la AF realizar. }\end{array}$ & $\begin{array}{l}\text { - Elaboración y } \\
\text { puesta en práctica } \\
\text { de calentamiento, } \\
\text { previo análisis de la } \\
\text { actividad física, de } \\
\text { forma autónoma y } \\
\text { prevención de } \\
\text { lesiones. }\end{array}$ \\
\hline & $\begin{array}{l}\text { - Cuales son } \\
\text { cualidades físicas- } \\
\text { salud. } \\
\text { - Acondicionamiento } \\
\text { CF salud. }\end{array}$ & $\begin{array}{l}\text { - Cualidades físicas- } \\
\text { salud y relación con } \\
\text { la salud. } \\
\text { - Resistencia y } \\
\text { flexibilidad. }\end{array}$ & $\begin{array}{l}\text { - Cualidades } \\
\text { físicas-salud y } \\
\text { relación con la } \\
\text { salud e incidencia } \\
\text { en el organismo. } \\
\text { - Sistemas de } \\
\text { entrenamiento de } \\
\text { resistencia, } \\
\text { flexibilidad, fuerza } \\
\text { general. }\end{array}$ & $\begin{array}{l}\text {-Sistemas y métodos } \\
\text { de entrenamiento, } \\
\text { cualidades físicas- } \\
\text { salud y efectos en la } \\
\text { salud. } \\
\text { - Elaboración y } \\
\text { puesta práctica plan } \\
\text { de trabajo mejorar } \\
\text { CF. }\end{array}$ \\
\hline & $\begin{array}{l}\text { - Adopción posturas } \\
\text { adecuadas en la AF y } \\
\text { vida cotidiana. } \\
\text { - Mejora musculatura } \\
\text { de sostén. }\end{array}$ & $\begin{array}{l}\text { - Valorar la } \\
\text { adopción de posturas } \\
\text { correctas. }\end{array}$ & $\begin{array}{l}\text { - Adoptar posturas } \\
\text { correctas durante } \\
\text { la práctica de AF. }\end{array}$ & $\begin{array}{l}\text { - Valorar y criticar } \\
\text { la adopción de } \\
\text { posturas correctas } \\
\text { en la actividad física } \\
\text { y vida cotidiana. }\end{array}$ \\
\hline & $\begin{array}{l}\text { - Higiene corporal tras } \\
\text { la EF. }\end{array}$ & $\begin{array}{l}\text { - Hidratación y } \\
\text { hábitos saludables. }\end{array}$ & $\begin{array}{l}\text { - Alimentación y } \\
\text { relación ingesta- } \\
\text { gasto, alimenta- } \\
\text { ción y salud. }\end{array}$ & $\begin{array}{l}\text { - Valorar y criticar } \\
\text { hábitos saludables. } \\
\text { - Primeros auxilios } \\
\text { en lesiones. }\end{array}$ \\
\hline $\begin{array}{c}\text { Bloque } \\
2 \\
\text { Juegos y } \\
\text { Deportes }\end{array}$ & $\begin{array}{l}\text { - Deporte como } \\
\text { fenómeno cultural. } \\
\text {-habilidades motrices. } \\
\text { - Deportes } \\
\text { individuales y } \\
\text { colectivos. } \\
\text { - Introducción reglas. } \\
\text { - relación salud. }\end{array}$ & $\begin{array}{l}\text { - Téc-Tac de un } \\
\text { deporte individual. } \\
\text { - Deportes } \\
\text { colectivos y de } \\
\text { adversario como } \\
\text { fenómeno social y } \\
\text { cultural. } \\
\text { - Colaboración. }\end{array}$ & $\begin{array}{l}\text { - Deportes } \\
\text { colectivos. }\end{array}$ & $\begin{array}{l}\text {-Deportes de } \\
\text { adversario con } \\
\text { implemento. } \\
\text {-Planificación de } \\
\text { torneos. } \\
\text { - Practicar. }\end{array}$ \\
\hline $\begin{array}{l}\text { Bloque } \\
3 \\
3 \\
\text { Expresión } \\
\text { Corporal }\end{array}$ & $\begin{array}{l}\text {-El cuerpo y el } \\
\text { movimiento. } \\
\text { - Conciencia corporal. } \\
\text { - desinhibición. }\end{array}$ & $\begin{array}{l}\text { - Dominio y control } \\
\text { corporal. } \\
\text { - Introducción base } \\
\text { rítmica } \\
\text {-Improvisaciones. }\end{array}$ & $\begin{array}{l}\text { - Bailes y danzas } \\
\text { ind-colectiva y por } \\
\text { parejas como parte } \\
\text { de la cultura. }\end{array}$ & $\begin{array}{l}\text {-Composiciones } \\
\text { coreográficas } \\
\text { utilizando diferentes } \\
\text { elementos. } \\
\text { - Creatividad. } \\
\text { - Aportación en } \\
\text { grupo. } \\
\end{array}$ \\
\hline $\begin{array}{c}\text { Bloque } \\
4 \\
\text { Actividades } \\
\text { en el Medio } \\
\text { Natural }\end{array}$ & $\begin{array}{l}\text {-Las actividades en el } \\
\text { medio natural. } \\
\text { - Realizar recorridos a } \\
\text { partir de la } \\
\text { identificación señales } \\
\text { de rastreo. } \\
\text {-Normas de } \\
\text { conservación del } \\
\text { medio. }\end{array}$ & $\begin{array}{l}\text { - Senderismo. } \\
\text { - Realizar recorridos } \\
\text { a partir de la } \\
\text { identificación } \\
\text { señales de rastreo de } \\
\text { forma autónoma. } \\
\text { - Valoración del } \\
\text { medio como uso } \\
\text { recreativo. }\end{array}$ & $\begin{array}{l}\text { - Orientación. } \\
\text { - Normas de } \\
\text { seguridad en las } \\
\text { actividades de } \\
\text { orientación. }\end{array}$ & $\begin{array}{l}\text { - Relación AFIN y } \\
\text { salud. } \\
\text { - Organización y } \\
\text { participación en } \\
\text { AFIN. } \\
\text { - Concienciarse de } \\
\text { la importancia de las } \\
\text { AFIN. }\end{array}$ \\
\hline
\end{tabular}


Tabla 3.- Contribución integrada de los contenidos del área de Educación Física al desarrollo de las competencias básicas.

\begin{tabular}{|c|c|c|}
\hline $\begin{array}{l}\text { Competencia } \\
\text { básica }\end{array}$ & Contenidos que contribuyen a su consecución & Bloques \\
\hline \multirow{9}{*}{$\begin{array}{l}\text { Competencia } \\
\text { en el } \\
\text { conocimiento y } \\
\text { la interacción } \\
\text { con el mundo } \\
\text { físico }\end{array}$} & $\begin{array}{l}\text { - Los juegos y deportes en la naturaleza y los contenidos actitudinales } \\
\text { asociados a los mismos. }\end{array}$ & B4 \\
\hline & - El juego con materiales reciclados y reutilizados. & B2 \\
\hline & $\begin{array}{l}\text { - La adquisición de hábitos saludables de ejercicio físico para la ocupación } \\
\text { del tiempo de ocio. }\end{array}$ & B1-2-3-4 \\
\hline & - La higiene, la corrección postural y la alimentación equilibrada. & B1 \\
\hline & - Riesgos inherentes a la práctica de ejercicio. & B1-2-3-4 \\
\hline & $\begin{array}{l}\text { - Riesgos asociados al sedentarismo, al consumo de sustancias tóxicas o al } \\
\text { abuso del ocio audiovisual. }\end{array}$ & B1 \\
\hline & - Conocimiento del deporte en relación al medio. & B1-2-3-4 \\
\hline & - Adecuada percepción de uno mismo y del espacio físico. & B1-2-3-4 \\
\hline & $\begin{array}{l}\text { - Interactuar con el espacio; moverse con él, orientarse y resolver problemas } \\
\text { en los intervengan los objetos y su posición. }\end{array}$ & B1-2-3-4 \\
\hline \multirow{10}{*}{$\begin{array}{l}\text { Competencia } \\
\text { social y } \\
\text { ciudadana } \\
\text { ayudando a } \\
\text { aprender a } \\
\text { convivir }\end{array}$} & $\begin{array}{l}\text { - Elaboración y aceptación de las reglas, respeto a la autonomía personal, } \\
\text { participación y valoración de la diversidad. }\end{array}$ & B1-2-3-4 \\
\hline & - Actividades dirigidas a la adquisición de las habilidades motrices. & B2-3 \\
\hline & - Actividades físicas competitivas. & B2 \\
\hline & $\begin{array}{l}\text { - Los juegos de conocimiento y presentación, los juegos de autoestima, } \\
\text { afectivos y de animación. }\end{array}$ & B3 \\
\hline & - Técnicas de relajación y visualización creativa. & B1 \\
\hline & $\begin{array}{l}\text { - Juegos y deportes cooperativos, remodelación de juegos competitivos } \\
\text { desde una óptica cooperativa, juegos de expresión corporal y de resolución } \\
\text { de conflictos. }\end{array}$ & B1-2-3-4 \\
\hline & - Juegos y danzas de Andalucía y de otras culturas y países. & B3 \\
\hline & - Recopilación de juegos tradicionales. & B2 \\
\hline & - Juegos y deportes alternativos. & B2 \\
\hline & $\begin{array}{l}\text { - Juegos con materiales reciclados y reutilizados, la construcción de } \\
\text { juguetes, juegos medioambientales, juegos tradicionales y las actividades } \\
\text { físicas en la naturaleza. }\end{array}$ & B2-4 \\
\hline \multirow{3}{*}{$\begin{array}{l}\text { Competencia } \\
\text { cultural y } \\
\text { artística }\end{array}$} & - La representación dramática, la música y la danza, el lenguaje corporal. & B3 \\
\hline & $\begin{array}{l}\text { - Preparación de montajes o producciones expresivas, representaciones } \\
\text { teatrales o bailes. }\end{array}$ & B3 \\
\hline & $\begin{array}{l}\text { - Deportes, juegos tradicionales, actividades expresivas, la danza y su } \\
\text { consideración como patrimonio de los pueblos. }\end{array}$ & B2-3 \\
\hline \multirow{3}{*}{$\begin{array}{l}\text { Competencia } \\
\text { relativa a la } \\
\text { autonomía e } \\
\text { iniciativa } \\
\text { personal }\end{array}$} & $\begin{array}{l}\text { - El esquema corporal, las habilidades perceptivo-motrices y las } \\
\text { coordinaciones. }\end{array}$ & B1-2-3 \\
\hline & - Calentamiento autónomo, organización de juego. & B1-2-3-4 \\
\hline & - Actuaciones frente a un público, las actividades de "riesgo". & B2-3-4 \\
\hline
\end{tabular}


De forma más concreta y precisa, encontramos también algunos cambios con respecto al Decreto anterior como las que a continuación se referencian:

$\square$ De $1^{\circ}$ a $2^{\circ}$ se debe impartir un deporte colectivo distinto al curso anterior.

$\square$ La expresión corporal hace mucho más referencia a la danza que a la dramatización.

$\square$ Educación permanente del cuidado e higiene corporal así como de la adopción de posturas correctas en la actividad física y en la vida cotidiana.

$\square$ Incorporación en todos los contenidos a aplicar el desarrollo de las nuevas tecnologías de la información. Así por ejemplo, en el Capítulo 1 de la LEA en artículo 7 refiere "el acceso a las tecnologías de la información y la comunicación en la práctica educativa y el uso seguro de Internet en los centros" como un derecho del alumnado, de ahí que lo tomemos en cuenta como uno de los recursos a utilizar en el aula y la contribución al desarrollo de la lectura.
Importancia de la transferencia de aprendizaje de contenidos para la adquisición y desarrollo de tareas más complejas.

$\square$ Contenidos secuenciados para conducir a la autonomía del alumnado, para que planifique y desarrolle su propia actividad física, tanto en el trabajo de las capacidades físicas como en la organización de forma recreativa de su ocio, invitándole al asociacionismo deportivo, sea o no de forma reglada.

$\square$ El desarrollo de los contenidos utilizando diferentes recursos didácticos aumenta el interés del alumnado y facilita el desarrollo de aprendizajes significativos.

$\square$ Los contenidos deberán atender a la diversidad de alumnado convirtiéndose en un aspecto característico de la práctica docente diaria. La LEA, en su artículo 113, Principio de equidad, habla de las "necesidades específicas de apoyo educativo aquel que presenta necesidades específicas...de orden físico, psíquico, cognitivo o sensorial, el que por proceder de otros países...”. De este modo, la aten-

Tabla 4.- Contribución integrada de los contenidos del área de Educación Física al desarrollo de las competencias básicas.

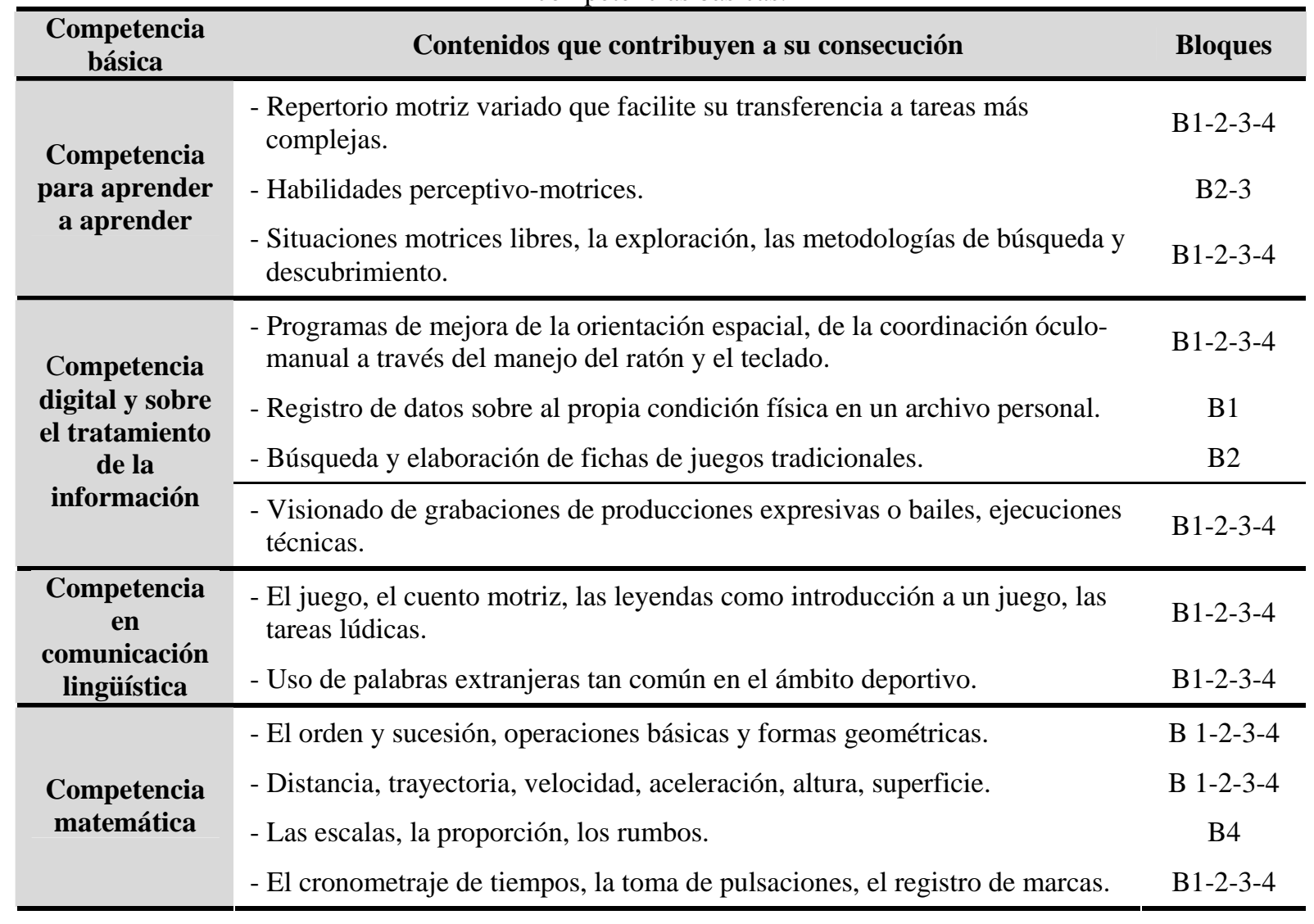


ción a la diversidad debe convertirse en un aspecto característico de la práctica docente diaria.

Y todo ello, sin perder de vista la realidad, es decir, utilizando actividades y unidades que ya hayan sido realizadas en el aula, habiendo comprobado su idoneidad, contextualizadas dentro del entorno y comprobado que su realización ayudan y complementan a conseguir los objetivos de nuestra materia desde el punto de vista tratado en la justificación de este mismo proyecto; lo que implica, además, trabajar de forma crítica, teniendo en cuenta la opinión que el alumnado tiene sobre nuestra propia materia, los diferentes objetivos que nos debemos plantear desde la misma, etc.

Obviamente, para todo ello va a ser necesario una comunicación, relación y compenetración continua y constante que va a verse facilitada por el uso de las nuevas tecnologías y la plataforma que proporciona el CEP Cuevas-Olula.

\section{Fases para el desarrollo del proyecto.}

a) Definición de los objetivos del proyecto.

b) Diseño del cuestionario para conocer la opinión el alumnado.

c) Aplicación del cuestionario a todo el alumnado de educación secundaria (población) de los centros implicados.

d) Tabulación de los datos obtenidos para ser introducidos en un paquete estadístico informático.

e) Análisis de los resultados obtenidos.

f) Secuenciación de los contenidos por curso en función de los resultados, teniendo en todo momento como referencia la normativa vigente.

g) Puesta en común de los aspectos que deben recoger cada una de las unidades didácticas.

h) Reparto de las unidades didácticas a elaborar por curso.

i) Elaboración de las unidades didácticas.

j) Puesta en común de los materiales curriculares elaborados.

k) Puesta en práctica de las unidades didáctica, previa contextualización al centro determinado.

l) Reuniones para resolver los diversos problemas que se hayan podido plantear a la hora de la puesta en práctica en un contexto concreto.

\section{5.- RESULTADOS ESPERADOS.}

Resulta conveniente terminar aludiendo al momento sociopolítico que nos toca vivir, también como docentes, ya que incide directamente en nuestra planificación didáctica. Este, se puede vivir como un momento de incertidumbre o de oportunidad. La oportunidad reside en reflexionar sobre la evolución de los últimos años y afrontar una etapa en la que aspectos como la atención a la diversidad, la adquisición de las competencias básicas o la programación de contenidos más allá de los conceptuales, adquieran mayor importancia, trabajando sobre la educación integral del alumnado.

Así presentamos, una propuesta fundamentada en el marco general en el que nos encontramos, que como docentes hemos concretado y con nuestras habilidades y recursos personales hemos intentado lograr la máxima coherencia, tratando de tener una lógica interna que va desde las intenciones hasta la evaluación y que será lo que dará solidez y validez a nuestro proyecto.

Teniendo en cuenta los datos de algunos estudios recientes (Thompson, Humbert y Mirwald, 2003; Trudeau y Shepherd, 2005), que sugieren que la calidad de los programas de Educación Física pueden contribuir de forma positiva a mantener posiciones inicialmente favorables hacia la actividad física, desde este proyecto se trata de conectar y partir de los intereses de nuestro alumnado, lo cual supone, además, una oportunidad para ofrecerles programas compuestos por una amplia gama de actividades, centradas en contenidos que se alejan de los tradicionalmente trabajados en esta asignatura. Además de una mayor conexión del alumnado con la Educación Física, incrementando el disfrute y el prendizaje, de esta manera, se incrementa la probabilidad, como expone Bocarro et al (2008) de que ese mayor número de experiencias deportivas tengan un impacto positivo en la adhesión a la actividad física a lo largo de toda su vida. Al menos éstas son las premisas expuestas por la teoría de repertorio del ocio (Iso-Ahola, Jackson y Dunn, 1994) que sugiere que las personas que desarrollan un espectro más amplio de actividades a una edad más temprana tienen más probabilidades de seguir participando en las 
actividades a medida que envejecen debido a un repertorio más amplio de prácticas de ocio.

\section{6.- REFERENCIAS BIBLIOGRÁFICAS.}

Arias, A. \& Fernández, B. (1998). La encuesta como técnica de investigación social. En A. J. Rojas, J. S. Fernández \& C. Pérez, Investigar mediante encuestas. Fundamentos teóricos y aspectos prácticos (pp. 31-44). Madrid: Síntesis.

Bocarro, J., Michael, A. K., Jonathan, C. \& Sott, F. (2008). School Physical Education, Extracurricular Sports, and Lifelong Active Living. Journal of Teaching in Physical Education, 27, 155-166.

Colás P. \& Buendía, L. (1994). Investigación educativa. Sevilla: Alfar.

Decreto 231/2007, de 31 de julio, por el que se establece la ordenación y las enseñanzas correspondientes a la educación secundaria obligatoria en Andalucía (BOJA núm. 156 de 8 de agosto de 2007).

Decreto 416/2008, de 22 de julio, por el que se establece la ordenación y las enseñanzas correspondientes al bachillerato en Andalucía (BOJA núm. 149 de 28 de julio de 2008).

European Heart Network (1999). Physical Activity and Cardiovascular Disease Prevention in the European Union [on line]. Disponible en: http://www.ehnheart.org/files/phyactivity084635A.pdf. [Consulta: 2008, 2 de septiembre].

European Heart Network (2001). Children and young people: the importance of physical activity [on line]. Disponible en: http://www.ehnheart.org/files/phyactivity084635A.pdf. [Consulta: 2008, 17 de agosto].

Flintoff, A. \& Scraton S. (2001). Stepping into Active Leisure? Young Women's Perceptions of Active Lifestyles and their Experiences of School Physical Education. Sport, Education and Society, 6(1), 5-21.

García Ferrando, M. (2000). La encuesta. En M. García Ferrando, J. Ibáñez, y F. Alvira (Eds.), El análisis de la realidad social. Métodos y técnicas de investigación (pp. 167-201) (3 $3^{\mathrm{a}}$ ed.). Madrid: Alianza editorial.

Hoffman, J. R., Kang, J., Faigenbaum, A. D., \& Ratamess, N. A. (2005). Recreational sports participation is associated with enhanced physical fitness in children. Research in Sports Medicine, 13, 149-161.

Iso-Ahola, S. E., Jackson, E. \& Dunn, E. (1994). Starting, ceasing, and replacing leisure activities over the life-span. Journal of Leisure Research, 26, 227-249.

Ley de Educación de Andalucía del 17/2007, de 10 de diciembre (BOJA núm. 252, de 26 de diciembre de 2007).

Ley Orgánica de Educación del 2/2006 de 3 de mayo (BOE num. 106, de 4 de mayo de 2006).

Luke, M. D. \& Sinclair, G. D. (1991). Gender Differences in Adolescents' Attitudes toward School Physical Education. Journal of Teaching in Physical Education, 11(1), 3146.

MacPhail, A., Kirk, D., \& Eley, D. (2003). Listening to young people's voices: Youth sports leaders' advice on facilitating participation in sport. European Physical Education Review, 9, 57-73.

Manzano, V. G., Rojas, A. J. \& Fernández, J. S. (1996). Manual para encuestadores. Fundamentos del trabajo de campo. Aspectos prácticos. Barcelona: Ariel.

Ministerio de Sanidad y Consumo. (2006). Encuesta Nacional de Salud de 2006 [on line]. Disponible en http://www.msc.es/estadEstudios/estadisticas lencuestaNacional/encuesta2006.htm [Consulta: 2008, 2 de noviembre].

Mowling, C. M., Brock, S. J., Eiler, K. K. \& Rudisill, M. E. (2004). Student motivation in physical education: breaking down barriers, Journal of Physical Education, Recreation and Dance, 75, 40-51.

Ortega, F. B., Ruiz, J. R, Castillo, M. J., Moreno, L. A., González-Gross, M., Wärnberg, J., Gutiérrez, A. y Grupo AVENA (2005). Bajo nivel de forma física en los adolescents españoles. Importancia para la salud cardiovascular futura (Estudio AVENA). Revista Española de Cardiología, 58(8), 898-909.

Padilla, J. L., González, A. \& Pérez, C. (1998). Elaboración del cuestionario. En A. J. Rojas, J. S. Fernández \& C. Pérez, Investigar mediante encuestas. Fundamentos teóricos y aspectos prácticos (pp. 115-140). Madrid: Síntesis. 
Piéron, M. (2005). Para una enseñanza eficaz de las actividades físico-deportivas (2 ${ }^{\mathrm{a}}$ ed.). Barcelona: Inde.

Real Decreto 1467/2007, de 2 de noviembre, por el que se establece la estructura del bachillerato y se fijan sus enseñanzas mínimas (BOE núm. 266 de 6 de noviembre de 2007).

Real Decreto 1631/2006, de 29 de diciembre, por el que se establecen las enseñanzas mínimas de la Educación Secundaria Obligatoria (BOE núm. 5, de 5 de enero de 2007).

Real Decreto 1631/2006, de 29 de diciembre, por el que se establecen las enseñanzas mínimas de la Educación Secundaria Obligatoria (BOE núm. 5 de 5 de enero de 2007).

Rikard, G. L. \& Banville, D. (2006). High school student attitudes about physical education. Sport, Education and Society, 11(4), 385400.

Serra, L., Ribas, L., Aranceta, J., Pérez, C., Saavedra, P. \& Pena L. (2003). Childhood and adolescent obesity in Spain. Results of the enKid study (1998-2000). Medicina Clínica, 121, 725-732.

Sierra Bravo, R. (2001). Técnicas de Investigación social. Teoría y ejercicios. Madrid: Paraninfo.
Taylor, W.C., Blair, S.N., Cummings, S.S., Wun, C.C., \& Malina, R.M. (1999). Childhood and adolescent physical activity patterns and adult physical activity. Medicine and Science in Sports and Exercise, 31(1), 118-123.

Telama, R., Yang, X., Viikari, J., Valimaki, I., Wanne, O. \& Raitakari O. (2005). Physical activity from childhood to adulthood: a 21year tracking study. American Journal of Preventive Medicine, 28, 267-273.

Thompson, A. M., Humbert, M. L., \& Mirwald, R. L. (2003). A longitudinal study of the impact of childhood and adolescent physical activity experiences on adult physical activity perceptions and behaviors. Qualitative Health Research, 13, 358-377.

Torre, E., Cárdenas, D., \& García Montes, E. (2001). Las percepciones que se derivan de las experiencias recibidas en las clases de educación física y su repercusión en los hábitos deportivos en el alumnado de bachillerato. Motricidad. European Journal of Human Movement, 7, 95-112.

Trudeau, F., \& Shepherd, R. J. (2005). Contribution of school programs to physical activity levels and attitudes in children and adults. Sports Medicine, 25, 89-105.

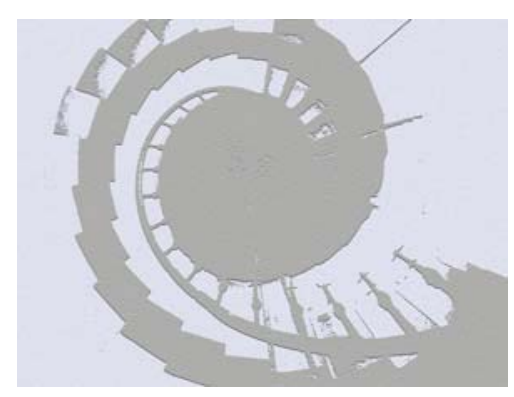

